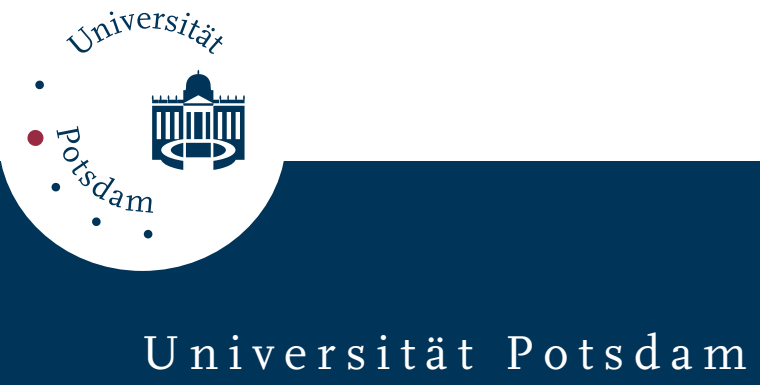

Margret Selting

\title{
Konstruktionen am Satzrand als interaktive Ressource in natürlichen Gesprächen
}

first published in:

Was determiniert Wortstellungsvariation? : Studien zu einem Interaktionsfeld von Grammatik, Pragmatik und Sprachtypologie / Brigitta Haftka (Hrsg.). Opladen : Westdt. Verl., 1994. ISBN 3-531-12490-0, S. 299-318

Postprint published at the Institutional Repository of the Potsdam University: In: Postprints der Universität Potsdam Philosophische Reihe ; 48 http://opus.kobv.de/ubp/volltexte/2010/4193/ http://nbn-resolving.de/urn:nbn:de:kobv:517-opus-41932

Postprints der Universität Potsdam Philosophische Reihe ; 48 


\title{
Konstruktionen am Satzrand als interaktive Ressource in natürlichen Gesprächen
}

\author{
Margret Selting
}

\section{Einleitung}

Ich möchte im Folgenden versuchen, formbezogen-systemlinguistische und konversations- und kontextualisierungsanalytische Fragen zu verbinden. Als Antwort auf die Frage dieses Sammelbandes, "Was determiniert Wortstellungsvariation?", möchte ich zeigen, daß grammatische Kategorien als interaktiv relevante Kategorien verwendet werden, die systematisch auf die Erfordernisse der Organisation der Interaktion zugeschnitten sind. Meine empirische Analyse basiert auf einem Datenkorpus natürlicher Gespräche. Konkret möchte ich mich mit syntaktischen Konstruktionen befassen, in denen Konstituenten vor oder nach einen syntaktisch vollständigen Satz gestellt werden, auf den sie morphologisch und/oder syntaktisch bezogen sind. Ich möchte folgende Punkte zeigen: (1) Die betreffenden Strukturen können nicht allein syntaktisch unterschieden werden, sondern Z.T. syntaktisch ambige Strukturen ergeben erst in ihrem Zusammenspiel mit der prosodischen Einheitenbildung unterschiedlich verwendete sprachliche Konstruktionen. (2) Diese so unterschiedenen sprachlichen Konstruktionen sind auf die Erfüllung unterschiedlicher interaktiver Funktionen in Gesprächen zugeschnitten.

Ich werde mich auf einige spezifische Typen von Voranstellungen vor und Nachstellungen nach Bezugssätzen beschränken, die sich gut für die Untersuchung des Zusammenspiels von Syntax und Prosodie eignen, nämlich diejenigen Herausstellungsstrukturen, die ALTMANN (1981) Linksversetzung (LV), Freies Thema (FT), Rechtsversetzung (RV) und z.T. Nachtrag (NT) genannt hat. Es handelt sich um Strukturen gesprochener Sprache, die z.T. allein prosodisch unterschieden werden. Ich werde zunächst Voranstellungen, dann Nachstellungen untersuchen, dabei jeweils zuerst die grammatische und prosodische Struktur dieser Konstruktionen analysieren, danach einige der Funktionen dieser Strukturen in der konversationellen Interaktion exemplifizieren.

Im Interaktionsmodell der Konversationsanalyse sind Sätze Turnkonstruktionseinheiten, mit deren Ende potentiell das Rederecht zur Disposition steht (SACKS/SCHEGLOFF/JEFFERSON 1974). Naturgemäß stellen sich damit am Anfang von Turns und Turnkonstruktionseinheiten andere Aufgaben als am Ende: am Anfang muß v.a. die Beziehung der Einheit zu Vorgängereinheiten signalisiert werden, am Ende muß die weitere Turnzuteilung geregelt werden. Auf die Lösung dieser Aufgaben sind die Konstruktionen am Satzanfang und am Satzende zugeschnitten. Das soll an einigen Beispielen exemplarisch verdeutlicht werden.

Die von mir präsentierten Beispiele für prosodisch unterschiedene Konstruktionen sind in den meisten Fällen, da prosodische Phănomene höchst relationalen Charakter haben, prototypische Beispiele. Dies impliziert, daß in manchen Fällen die vom Sprecher realisierten Formen nicht klar zuordbar und daher deren Interpretation und interaktive Bedeutung in der Interaktion aushandelbar sind. Dennoch können prototypische Pole an den Rändern der Kontinua unterschieden und genauer untersucht werden. Ich gehe davon aus, daß syntaktisch und/oder prosodisch unterschiedene Konstruktionstypen so sind, wie sie sind, weil sie auf unterschiedliche Funktionen in der Alltagskommunikation zugeschnitten und somit interaktiv motiviert sind. 


\section{Struktur und Funktion der Herausstellungen}

Die Herausstellungen müssen nach Voranstellungen vor den Satz und Nachstellungen nach dem Satz unterschieden werden. Dabei setze ich hier voraus, daß trotz erheblicher Definitionsprobleme (siehe zuletzt hierzu HofFMANN 1992), die Einheit des 'Satzes' bzw. des 'syntaktisch möglichen Satzes' in den hier zu behandelnden Konstruktionstypen identifiziert werden kann. Es geht mir v.a. um Konstruktionen, in denen eine Konstituente vor oder nach dem Satz angeordnet ist, die eine koreferente Pro-Form oder eine Bezugskonstituente innerhalb des Satzes vorweg- oder wiederaufnimmt. Herausstellung und Pro- bzw. Bezugs-Element sind semantisch und morpho-syntaktisch aufeinander bezogen. ALTMANN (1981) nannte die mich interessierenden Strukturen LV, FT und RV. Konstruktionen dieses Typs wurden bislang meistens anhand syntaktischer und morphologischer Kriterien unterschieden, der Verweis auf die Prosodie war jedoch immer schon zwecks Disambiguierung ansonsten formidentischer Konstruktionen nötig. ALTMANN (1981) geht ebenfalls von der Syntax aus, nimmt dennoch aber die Prosodie bzw. Intonation als konstitutives Merkmal der Strukturtypen hinzu; ähnlich geht AUER 1991 im Falle der Rechtsexpansionen vor, orientiert sich aber eindeutiger an empirischen Daten der mündlichen gesprochenen Sprache.

Jedoch können weder syntaktische noch morphologische Kriterien eindeutig zwischen z.B. den Konstruktionstypen LV und FT, und verschiedene Formen von RV unterscheiden. Deshalb muß m.E. die Prosodie sogar als primär differenzierendes Merkmal der Morphologie und Syntax übergeordnet werden. Dies entspricht einer einfachen Logik: Die Prosodie signalisiert die interaktiv relevante Einheitenbildung; erst auf der Grundlage dieser Segmentierung in Einheiten kann dann weiter nach den morpho-syntaktisch und anders signalisierten Beziehungen innerhalb von Einheiten und zwischen Einheiten gefragt werden. Eine solche Analyse anhand zunächst prosodischer und erst nachgeordnet morpho-syntaktischer Kriterien erlaubt eine formbezogene Klassifikation, die auch durch eine funktionale Analyse gestützt wird. Wie also ein syntaktisch u.U. ambiges Syntagma prosodisch segmentiert wird, hat interaktive Relevanz und Bedeutung.

Die untersuchten Konstruktionen müssen nach ihrer prosodischen Integration bzw. Selbständigkeit gegenüber dem Satz unterschieden werden. 'Prosodische Integration' wird durch Realisierung quasi "unter dem Dach" der ununterbrochen fortgesetzten Intonationskontur signalisiert; für 'prosodische Selbständigkeit' wird mithilfe eines Neuansatzes prosodisch eine neue Einheit hergestellt. Die Länge einer intern kohäsiven Intonationskontur wird in der Intonationszeile der Transkripte durch die Position der runden Klammern angezeigt. In den Transkripten stehen die Herausstellungen und deren Bezugsausdrücke kursiv. Bei der Analyse werde ich aus Platzgründen das anhand der Analyse zu rekonstruierende Merkmalsbündel der Struktur jeweils bereits vorgreifend anführen. 


\subsection{Voranstellungen vor den Satz: $L V$ und FT²}

(a) Linksversetzung

[+ prosodisch integriert]

[+ syntaktische Wiederaufnahme: XP - Pro-Form im Vorfeld]

[+ morphologische Übereinstimmung zwischen XP und Wiederaufnahme]

Bei der LV wird eine dem Satz vorangestellte Konstituente im Vorfeld des Folgesatzes mit einer koreferenten Pro-Form wiederaufgenommen; die Linksversetzung ist prosodisch in die Einheit integriert. Beispiele sind unter (1) - (7) zusammengestell:

(1) $\mathrm{K} 1: 828$

die MEIstn_, die woHN_, hier n paar wochen . H( /

)

(2) $\mathrm{K4}: 95$

meine nutter de macht zun beispiel so SAchen daß dann: $\mathrm{M} / /$

(3) $\mathrm{k} 2: 58$

un . die LEHrer die sAßen da alle auch .

M()

un so größere TIsche herum

(4) K1: 788

nämich ne FrEUNdin von mir die is auf eim *o . äh ohr TAUB

< all $\mathrm{M}(\mathrm{l}$

(5) Ko: 395

in dem BUCH da war das so.

$\mathrm{F}()$

daß die Leute irgdwann annem BLusturz gestorben sind

(6) $\mathrm{K} 1: 479$

und DREImal in der woche das REICHT dann $\mathrm{mh}$ :

(7) $\mathrm{K} 1: 1132 \mathrm{f}$.

nee so sTäNdich m JEmandn so um sich habm das köNNT ich AU nich

I $\mathrm{F}(\mathrm{l}$

Die LV kann jede beliebige Konstituente sein: NP wie in (1)-(4), PP wie in (5), AdVP wie in (6), VP wie in (7), usw. Auf die Linksversetzung folgt unmittelbar und ohne Pause oder prosodischen Bruch die Wiederaufnahme im Vorfeld des Verb-Zweit-Satzes. ${ }^{3}$ LV und Pro-Form können beliebigen Kasus haben; sie stimmen soweit wie möglich in ihren morphologischen Merkmalen für Kasus, Genus und Numerus überein. Die LV erhält einen steigenden oder fallenden Akzent, die Wiederaufnahme bleibt unakzentuiert. Aufgrund der Tatsache, daß diese LVen prosodisch in die Einheit integriert sind, fallen sie auditiv kaum als herausgestellte Elemente aut. Die LV beginnt eine ganz "normale" Kontur. 
LV plus Wiederaufnahme erscheinen wie eine doppelte Vorfeldbesetzung (vgl. hierzu auch ALTMANNS 1981 empirische und CARDINALETTIS 1987 kompetenztheoretische Analyse im Rahmen der generativen Grammatik).

Die LV ist eine markierte Form der Topikalisierung, wobei innerhalb einer prosodischen Einheit mit mindestens zwei Akzentdomänen ein mehrteiliger Fokus signalisiert wird (vgl. dazu UHMANN 1991): Ein Fokusteil wird erzeugt durch die Akzentuierung der LV selbst, ein zweiter durch die Ak zentuierung eines Elements der folgenden Prädikation. Beide Fokusteile sind rhematisch bzw. relevant gesetzt als Ausgangspunkte der damit eingeleiteten thematischen Sequenz. Zumindest in einigen Fällen sind LV-Konstruktionen dabei offensichtlich auch gut geeignet, Akzenthäufungen auf benachbarten Elementen durch die Einschiebung einer unakzentuierten Silbe zu verhindern (vgl. die Beispiele (1) und (3)).
(b) 'Freies Thema'
[-prosodisch integriert]
[+ syntaktische Wiederaufnahme: XP - beliebige Wiederaufnahme]
[ \pm morphologische Übereinstimmung zwischen XP und Wiederaufnahme]

Beim FT wird dem eigentlichen Satz eine Konstituente in eigener prosodischer Einheit vorangestellt und im Nachfolgesatz wiederaufgenommen. Das FT ist gegenüber dem Nachfolgesatz freier und selbständiger als die LV. Beispiele sind unter (8) - (14) zusammengestellt:

(8) $\mathrm{k2}: 693$

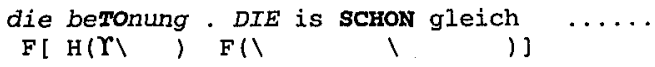

(9) K1: $130 f f$

diese ganzen FRAUnthemen von wegen emanzipaTION un so ...

<all all> M(l I /

die wern von den MEIsten fraun DIE sich damit beschäftigen <all all $>M$ (

und die da so: HINterstehn ...

irgnwie so HOCHgejubelt und, mh auch so exrRom ausgebreitet ne
$F(1)$

(10) K1: $35 \mathrm{ff}$

also ne. H(l)

I professorin von HaMburch

T(1)

un die is alle vierzehn TAge gekonm das war schon mal BLÖD
<all all>
$\mathrm{s}(1$
1) 
(11) $\mathrm{x} 1: 1043 \mathrm{f}$

also wOchenendfreundschaftn=

$\mathrm{M}$ ()

)

$=$ ICH hab das jetzt proBIERT zwei monate. $\mathrm{M} / \mathrm{C}$

<all?>

(12) K2: $327 \mathrm{ff}$

und gesang

$\mathrm{M}\left(/^{\prime}\right)$

$\underset{F(1}{\text { HABT ihr denn hier irgndwie son: son LEHrer oder wie LÄUFT das }}$

(13) $\mathrm{k} 3$ :

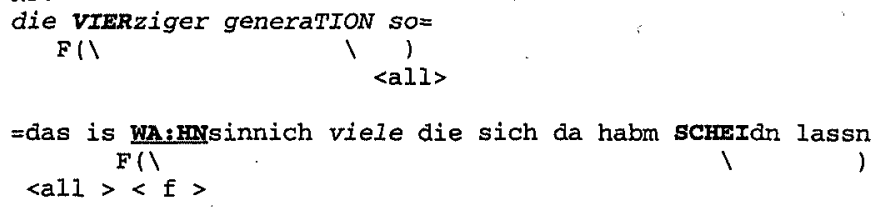

die VIERziger generaTION SO=

$F()$

$1<$ <ali $>$

=das is WA:HRinnich viele die sich da habm scheIdn lassn F(

$<$ all $><\mathrm{f}>$

(14) K2: $668 \mathrm{f}$

un mit meiner ARbeit. DIE hab ich hier as is fest

$\mathrm{M}(/) \mathrm{M}(/)$ )

Das FT steht in eigener prosodischer Einheit, hat meist einen oder mehrere fallende oder steigende Akzente und ist durch einen Neuansatz und ggf. eine Pause vom Satz abgetrennt. In meinem Korpus kommen nur NPn und PPn als FT vor; sie werden in unterschiedlicher Weise wiederaufgenommen. Zwischen dem FT und der Wiederaufnahme muß keine morphologische Übereinstimmung bestehen, aber es kann durchaus morphologische Übereinstimmung bestehen.

Die ersten Beispiele, (8) - (10), ähneln noch stark den LV, da auch hier die vorangestellten Konstituenten im Vorfeld des Folgesatzes wiederaufgenommen werden. Doch wird in (8) die wiederaulnehmende Pro-Form DIE akzentuiert. In (9) wird das FT expandiert, bevor es im Vorfeld des Nachfolgesatzes wiederaufgenommen wird. In (10) wird zum einen das FT expandiert und dabei präzisiert, zum anderen tritt auch noch der Konnektor un zwischen die Voranstellung und den. Folgesatz.

Demgegenüber werden die FT in den Beispielen (11) - (14) auch innerhalb der Folgesätze erst an späterer Stelle wiederaufgenommen, und zwar in (11) - (12) durch die morphologisch nicht übereinstimmende unspezifischere Pro-Form das, in (13) durch viele. (14) ist ein Beispiel, das nach gängigen Kriterien nicht unbedingt als FT akzeptiert würde, weil die Voranstellung nicht im Nominativ steht. Da aber m.E. eine Kasusbeschränkung der FT auf den Nominativ sowieso nicht hinreichend zwischen FT und LV unterscheidet und deshalb diese Kasusbeschränkung keine Analysevorteile er- 
bringt, umgekehrt aber Konstruktionen wie in (14) ebenso wie Nominativ-FT in Folgesatz wiederaufgenommen werden, analysiere ich sie ebenfalls als FT (siehe hierzu genauer SELTING 1993b).

Generell wird das FT im Nachfolgesatz an irgendeiner Stelle in irgendeiner Weise wiederaufgenommen. Das FT kann durch Partikeln, Anhängsel, Relativsätze o.ä. expandiert werden; zwischen das FT und den Nachfolgesatz können weiterhin z.B. Parenthesen eingeschoben werden. ${ }^{4}$ Konnektoren und Gliederungssignale können sowohl vor das FT als auch vor den Nachfolgesatz treten. Das FT kann zwar - wie eine LV - im Vorfeld wiederaufgenommen werden, wird dann aber - im Unterschied zur Linksversetzung - deutlich prosodisch abgesetzt und zudem wird die Vorfeldkonstituente gegebenenfalls erneut akzentuiert.

Diese Eigenschaften des FT zeigen folgendes: Es kann sich im Unterschied zur LV auf keinen Fall um eine doppelte Vorfeldbesetzung handeln, sondern es ist eine gegenüber dem Satz selbständigere Voranstellung. Das FT ist eine vorangestellte eigenständige Konstituente, die im Folgesatz wiederaufgenommen wird (vgl. auch ALTMANN 1981 und CARDINALETTI 1987).

Wenn das FT in eigenständiger prosodischer Einheit einen Akzent hat, erzeugt es einen semantischen Fokus. Unabhängig davon, ob im Folgesatz mit Hilfe eines oder mehrerer Akzent domänen ein ein- oder mehrteiliger Fokus signalisiert wird, konstituiert hier also das FT einen eigenständigen semantischen Fokus vor dem Satz.

\subsection{Funktionen der Voranstellungen}

Die Strukturanalyse der Voranstellungen hat ergeben, daß es sich bei Linksversetzungen um doppelte Vorfeldbesetzungen und die Herstellung eines mehrteiligen Fokus und bei den Freien Themen um vorangestellte eigenständige Konstituenten mit eigenem semantischen Fokus vor dem Satz handelt. Wozu gebraucht der Sprecher diese Konstruktionen?

Ebenso wie bei der einfachen Topikalisierung scheinen auch die markierteren Voranstellungen Sachverhalte zu topikalisieren, über die dann im Folgesatz und -gespräch weitere Ausführungen gemacht werden. ${ }^{5}$ Bei beiden Konstruktionen bleibt der so topikalisierte Gesprächsgegenstand weiterhin thematisch. Um zu exemplifizieren, was die unterschiedlichen Konstruktionen je Unterschiedliches leisten, betrachte ich nunfolgend an zwei Beispielen ihre offenbar den Erwartungen entsprechende Verwendung und Handhabung in ihren vorangehenden und nachfolgenden Gesprächskontexten; auf "Ausnahmen" kann ich aus Platzgründen nicht eingehen. - Die für die Analyse unmittelbar relevanten Zeilen der folgenden Transkripte sind mit einem Sternchen versehen.

\section{(a) Linksversetzungen:}

Das Beispiel (15) zeigt eine Linksversetzung in ihrem Gesprächskontext:

\section{(15) $\times 3$ :}

453 I: das isja das xomische ne.

454 ich mein e vielleich KOMMT man da nich drumRuM ... 


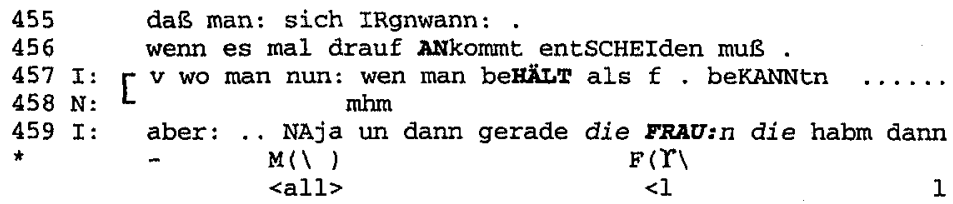

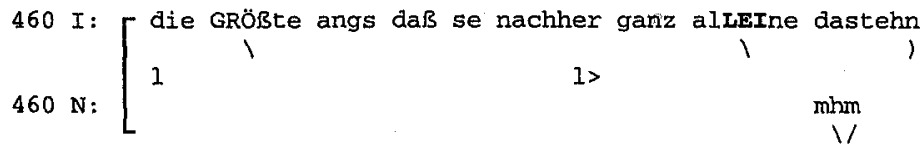

461 I: $[$ Gavz alleine ne

$462 \mathrm{~N}:[\mathrm{mhm} \ldots j$ ja as STIMMT

463 das wahrschein au noch mit n GRUND ......

464 wobei WENN frauen das geschafft habm... äh: ((usw.))

In der Einheit mit der LV in Zeile 459 kommt die LV, wie es oft der Fall ist, in Kookkurrenz mit Konstruktionen vor, in denen das vorherige Gesprächsthema unmittelbar fortgeführt wird und diese lokal anknüpfende kohärente Weiterführung des vorangegangenen Gesprächsthemas auch lexikalisch explizit signalisiert wird: mit der Anknüpfung aber: .. NAja un dann gerade die FRAUN wird das vorherige Thema der Trennungsprobleme bei Paaren durch Eingrenzung auf einen Partner fortgesetzt. Das mit der LV topikalisierte Thema Frauen und deren Umgang mit Ehetrennungen wird dann im Weiteren fortgefühit.

Diese Verwendungsweise der LV stimmt überein mit meinem in SELTING 1993b ausführlicher dargestellten Analyseergebnis, daß mit der LV eine an den unmittelbaren Vorgängerkontext lokal kohärent anknüpfende und weiterführende Konstituente topikalisiert wird. Mit der LV wird ein zuvor vom Sprecher selbst oder vom Rezipienten etabliertes Gesprächsthema direkt weitergeführt. Ein weiterer Sachverhalt, ein weiteres (Beleg-) Beispiel, ein weiterer Aspekt für das bisherige und weitergeführte Gesprächsthema wird topikalisiert, bevor hierzu weitere Ausführungen gemacht werden. Es wird eine lokal anknüpfende Weiterführung des bisherigen Gesprächsthemas signalisiert (vgl. auch DURANTIOCHS 1979). Gegenüber der einfachen Topikalisierung handelt es sich um eine zusätzliche Fokussierung eines Sachverhalts, die zugleich als lokal kohärente Weiterführung und als Verschiebung des bisherigen Gesprächsthemas dargestellt wird. Entsprechend wird die Konstruktion mit Linksversetzung oft mitten in einem Turn verwendet.

(b) Freie Themen:

Eine Verwendungsweise des Freien Themas zeigt (16):

(16) K0:

$851 \mathrm{E}$ : [ FINdeste unter DEUtschn_, bestimmt öFter

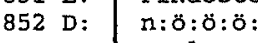

$853 \mathrm{M}$ : . was das SCHMUDdelige

$854 \mathrm{E}:$ nein. den MANgelnden LEBM,, Smut 


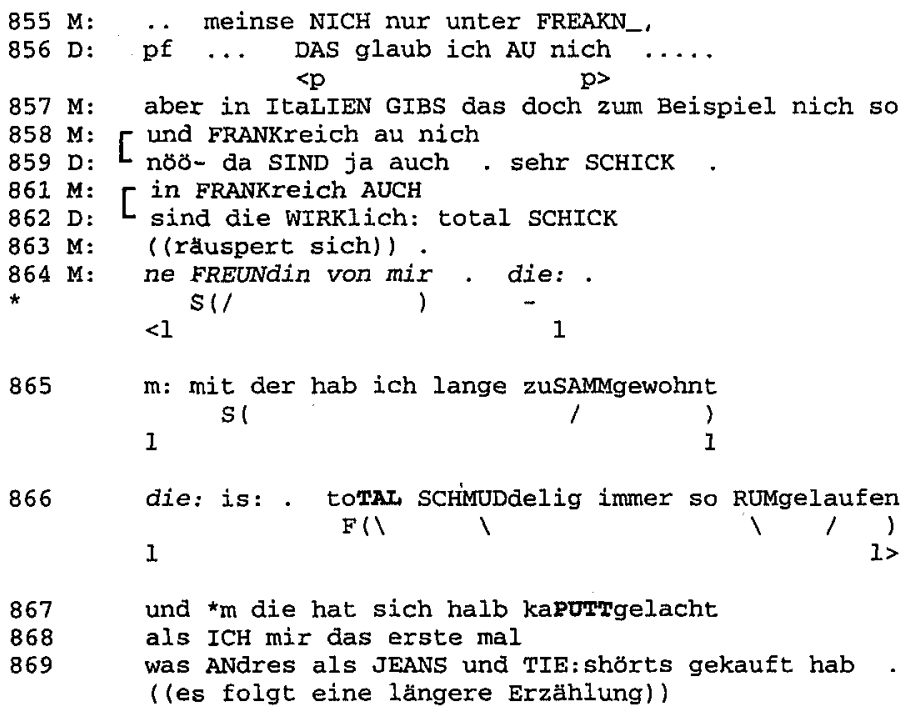

Wenn die LV eine lokale Anknüptung und direkte Weiterführung des bisherigen Gesprächs nahelegt, dann legt das FT gerade keine lokale und direkte Weiterführung nahe. Das FT knüpft nicht lokal an den unmittelbaren Vorgängerkontext an, sondern ist im Rahmen eines übergeordneten Gesprächsthemas kohärent. Im vorstehenden Beispiel beginnt $M$ mit der Fokussierung auf eine Freundin in Zeile 864 eine ausführliche Erzählung über die Wandlung ihrer Freundin durch einen Aufenthalt in Frankreich von einer schlampig zu einer sehr modisch sich kleidenden Frau. Der Punkt der Erzählung, daß genau der Frankreichaufenthalt eine solche Wandlung bewirkt hat, ist hier kohärent im Rahmen des Gesprächsthemas 'junge Leute und deren kulturspezifische Art sich zu kleiden', wird aber in der Erzählung selbst erst an späterer Stelle deutlich.

Insgesamt kommen FT überwiegend in anderen lokalen Gesprächskontexten vor als LV. Ca. Zwei Drittel aller FT in meinen Daten werden verwendet, entweder nachdem das Gespräch über das bisherige Gesprächsthema zu themenbeendenden allgemeinen Bemerkungen und ggf. auch längeren Pausen ohne einen "rechtzeitigen" und unproblematischen Sprecherwechsel geführt hat, oder nachdem aus anderen Gründen das vorherige Gespräch gestört worden war. Hier setzt das FT also einen Neubeginn nach einer "Flaute" oder Störung. In den anderen Fällen fokussiert das FT einen neuen Themenaspekt innerhalb eines übergeordneten Gesprächsthemas.

Das FT mit seinem eigenen semantischen Fokus erzeugt eine vorgreifende Aufmerksamkeitsfokussierung auf einen neuen oder erneut fokussierten Themenaspekt; es wird eine deutliche Fokussierung auf einen neuen thematischen Ausgangspunkt für die Fortsetzung des Gesprächs vorgenommen, eine neue thematische Richtung für die Weiterentwicklung des Gesprächs angegeben (vgl. OCHS/SCHIEFFELIN 1983). 
Rezipienten verstehen die Formulierung eines FT i.d.R. zugleich als Projektierung eines Nachfolgesatzes. Sprecher können sich nach der Produktion des FT sogar Pausen erlauben, ohne den Verlust des Rederechts zu riskieren. - Dann ist aber auch einsichtig, daß mithilfe des FT nach "Flauten", problematischen Sequenzen oder Themenbeendigungen der Turn übernommen werden kann, ohne daß der Sprecher seinen Folgeturn schon geplant haben muß. Die nach dem FT mögliche Pause erlaubt es ihm, den Nachfolgesatz zu verzögern und ggf. noch genauer zu planen. Zugleich könnte der Rezipient nach den FT prinzipiell durchaus noch eingreifen. Strukturell wäre hier die Voraussetzung gegeben für ein kooperatives Verfahren der Gesprächsweiterentwicklung. Aufgrund der anderen prosodischen Struktur und damit verbundenen zeitlichen Organisation ist die LV nicht in dieser Weise verwendbar.

\subsection{Nachstellungen nach dem Satz: "echte" RV und RV-Nachtrag}

Rechtsexpansionen sind ausführlich von AUER (1991) analysiert worden. Ich will eine solche Analyse hier nicht wiederholen, sondern mich nur auf einige Beispiele beschränken, um auch anhand der Nachstellungen nach dem Satz die Hypothese zu entwickeln, daß auch bei diesen Kon struktionen die Prosodie der Syntax übergeordnet werden muß, und daß auch hier die so unterschiedenen Konstruktionen ais interaktiv relevante Ressource verwendet werden. Ich behandele nur zwei Konstruktionstypen: die "echte" und die nachgetragene Rechtsversetzung (RV).

Bei der AV wird nach einem syntaktisch vollständigen Satz eine XP produziert, die eine koreferente Pro-Form im Vorgängersatz wiederaufnimmt. Diese syntaktische Struktur kann prototypisch aut zwei Weisen prosodisch organisiert werden: Die RV kann prosodisch integriert werden in die Vorgängerkontur, oder sie kann in neuer, prosodisch selbständiger Einheit nach dem Vorgângersatz stehen. Ich nenne diese beiden Typen "echte" RV' und 'RV-Nachtrag'. Da "echte" RV und RV-Nachtrag morpho-syntaktisch identisch sein können, differenziert u.U. nur die Prosodie zwischen diesen beiden Konstruktionen (vgl. auch AuER 1991). In Analogie zur Unterscheidung zwischen LV und FT müßte man auch hier von unterschiedlichen Konstruktionen sprechen, bei denen die Prosodie eine unterschiedliche Beziehung zum Bezugssatz anzeigt. ${ }^{6}$

(a) "Echte" Rechtsversetzung:

[+ prosodisch integriert]

[+ syntaktische Wiederaufnahme PRO - XP]

[+ morphologische Übereinstimmung zwischen PRO und XP]

Bei der prosodisch integrierten Form sind RV und koreferente Pro-Form eng aufeinander bezogen und weisen soweit wie möglich morphologische Kasus-, Numerus- und Genusübereinstimmung auf. Dies zeigen die folgenden Beispiele: 
(17) $\mathrm{KO}: 393$

wieso is der ANgenehm der tod

Ti

(18) $\mathrm{Kl}: 89$

DIE is auch NIE: WIEdergekomm die FRAU ,. TI

$<$ all >

(19) $\mathrm{K} 1: 565$

als ich das genörr hab daß du aus wilhelmshavm bist .. M()

(20) K5: $703 \mathrm{f}$.

WAS is eigentlich so

$F(/$

schwrarich daran an dieser STIFtungsgründung

1

(21) Ko: $626 \mathrm{ff}$

was MACHT die: die christine da ADCH mit mit dem SPIELN

$\mathrm{S}, \mathrm{H}(\mathrm{C}$

(22) K2: $375 f$.

ich BIN auch: , zum beispiel

F()

$<a 11>\langle d>$

relativ SpR̈ damit angefangn mit . mit sINgen

(23) K2: 308

also mir EäLLT das sehr schWER .. das spieln F(I

$<\mathrm{p} \quad \mathrm{p}\rangle$

Hier sehen wir als RV-Ausdrücke eine NP in (17), (18) und (23), eine CP in (19), eine PP in (20) (22). Innerhalb einer solchen prosodisch integrierten Einheit ist offenbar nichts zwischen Satz und folgender RV einschiebbar. Wenn hier Verzögerungspausen entstehen, wie in (22) und (23), so werden die Fortsetzungen der Einheit nach der Pause als Fortsetzungen kontextualisiert: In (22) geht der Sprecher nach dem Satz zunächst mit dem ersten mit in die RV hinein, bevor er eine Pause macht; nach der Pause wiederholt er die Präposition mit in derselben Tonhöhe und Lautstärke wie vor der Pause und kennzeichnet damit die Fortsetzung der Einheit als Fortsetzung der Einheit. In (23) führt der Sprecher ebenfalls nach der Pause mit der RV den bisherigen Tonhöhenverlauf ohne Tonhöhenbruch, aber mit geringerer Lautstärke, einfach fort und signalisiert damit eine Fortsetzung der Einheit, keinen Neuansatz; retrospektiv wird die Pause also als einheiten-interne und die Fortsetzung als ein 'Auslaufen' kontextualisiert. In meinem Korpus werden von 16 Beispielen 13 ohne und 3 mit einer einheiten-internen Pause konstruiert. Aus der engen Beziehung zwischen Satz und prosodisch integrierter RV könnte man schließen, daß es sich syntaktisch um eine Adjunktion an den Vorgängersatz handelt (vgl. auch ALTMANN 1981: 54ff.). 
Die AV kann einen Akzent erhalten, wie in (18) und (20) - (22), oder auch unakzentuiert bleiben wie in (17), (19) und (23). Obwohl die Akzente auf den RV keine primären sind und 2.T. auch wie rhythmisch motivierte Akzente erscheinen, erzeugen sie eine weitere Akzentdomäne und konstituieren damit einen (weiteren) Teil eines mehrteiligen Fokus gegen Ende der Einheit. Diese akzentuierten RV ähneln auch Konstruktionen wie Ausklammerungen (vgl. v.a. (20)), wohingegen die unakzentuierten RV auch einfachen Einheiten- und Satzverlängerungen mit optionalen Elementen ähneln (Vgl. SELTING 1991).

(b) Rechtsversetzungs-Nachträge

[- prosodisch integriert]

[+ syntaktische Wiederautnahme PRO - XP]

[ \pm morphologische Übereinstimmung zwischen PRO und XP]

Nachträge bilden prosodisch selbständige Einheiten. Șie sind laut Altmann 1981 elliptische Ausdrücke, die sich auf einen Bezugsausdruck im Vorgängersatz beziehen. Der Nachtragsausdruck ist morpho-syntaktisch wie der Bezugsausdruck markiert und mit Bezug auf den Satzrahmen des Vorgängersatzes zu interpretieren. Der Nachtrag hat i.d.R. eine Akzenttonhöhenbewegung; diese wiederholt die letzte Akzenttonhöhenbewegung des Vorgängersatzes (vgl. auch SELTING 1991).

Bei nachgetragener RV wird die RV in neuer, selbständiger prosodischer Einheit formuliert, oft nach einer Pause:

(24) K1: $880 \mathrm{f}$

R: also Do minst jetz. leute von der uns ne .

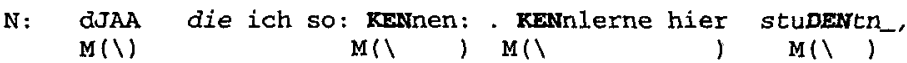

(25) K2: $478 f$

aso IRgnđwann: muß ich das bestimmt AU noch mal machen $\mathrm{M}(\mathrm{I}$

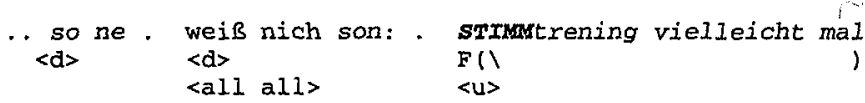

(26) K1: $279 f$

in musIK zum beispiel is es ganz ANders ne $\mathrm{H}, \mathrm{F}(\mathrm{l}$

()

.. die zuSAMM_, setzung
M(

(27) $\mathrm{K} 1$ : $311 \mathrm{f}$

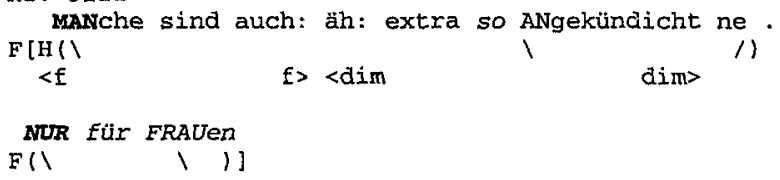


(28) K2: 468ff

R: =aber (0.6) ich: WILL mix das auch offenhaltn so ne $M()$

(29) K4: 734ff

734 L: =die Kom mir immer melancholisch vor ne

$\mathrm{H}, \mathrm{F}$ ()

$735 \mathrm{E}:$ die stuDENIN
M()

736 L: iJA .. weil ich GLAUbe nichr M(I) H(I)

In einem RV-Nachtrag können offenbar sämtliche Pro-Elemente eines Satzes expliziert und damit der syntaktisch vollständige Satz expandiert werden. Auch bei diesen Beispielen stimmen die RVAusdrücke mit Pro-Formen im Vorgängersatz überein, wir haben NPn in (24) - (26) und (29), eine AP in (27) und eine infinite Phrase in (28). In (25) und (26) ist die morphologische Übereinstimmung zwischen das bzw. es und der Nachtrags-RV son: STIMMtrening bzw. die zuSAMMsetzung infolge der Wahl der generelleren Pro-Formen nicht so eng wie in den übrigen Beispielen, dennoch ähneln die Beispiele noch eher den RV als anderen Nachtragstypen. In (29) wird der RV-Nachtrag von der Rezipientin des Satzes formuliert.

Hier stehen die RV-Ausdrücke in einer eigenen prosodischen Einheit nach dem Vorgängersatz. Zudem finden wir Einschübe zwischen dem Satz und dem RV-Ausdruck, nämlich Abgrenzungspausen (siehe (25) - (28)) und/oder Anhängsel-Tags wie ne nach dem Satz (siehe (26) (29)); in (25) formuliert eine Sprecherin eine Zwischenbemerkung innerhalb der Nachtrags-RV; in (28) finden wir weiterhin auch noch eine Rezipientenreaktion, bevor R einen RV-Ausdruck mit Bezug auf den Vorgängersatz formuliert; in (29) findet zwischen Vorgängersatz und RV-Nachtrag ein Sprecherwechsel statt.

Ebenso wie Einschübe zwischen dem Satz und dem FT legen auch diese Einschübe zwischen Satz und nachgetragener RV nahe, daß die nachgetragene RV in lockererer Beziehung zum Satz steht als die prosodisch integrierte RV. Sie hat starke Ähnlichkeit mit anderen Nachträgen. Diese nachgetragenen RV sind nicht als Adjunktionen an den Satz formuliert, sondern als eigenständige Konstituenten, die durch ihre morphologische Markierung ihre kohäsive Beziehung zum Vorgängersatz ausdrücken.

Auch die prosodisch selbständig nachgetragene RV kann sowohl einen Akzent haben (alle Beispiele außer (28)), als auch unakzentuiert bleiben ((28)). Wie beim FT, erzeugt auch hier die Akzentuierung des Nachtrags einen eigenen semantischen Fokus. Auch hier ist die Hypothese plausibel, daß aufgrund ihrer anderen prosodischen und zeitlichen Organisation die Konstruktion des RVNachtrags für andere interaktive Aufgaben als Lösung geeignet ist als die prosodisch integrierte "echle" RV. 


\subsection{Funktionen der Nachstellungen}

AUERS (1991) Fazit aus seiner Analyse von Rechtsexpansionen ist folgendes: Wenn davon ausgegangen wird, daß im Gespräch mögliche Abschlußpunkte syntaktischer Konstruktionen immer auch Stellen möglicher Turnübergabe sind, dann "ist jede solche Expansion von seiten des augenblicklichen Sprechers gefährdet, in Konkurrenz treten zu müssen mit einer Äußerung eines anderen Teilnehmers" (ebd.: 152). "Expansionen bilden also nicht nur den Rand eines Satzes, sie sind auch der Rand des Terrains, auf dem der Sprecher uneingeschränkt 'das Sagen hat'" (ebd.: 153). AUER bringt derartige Expansionen in Zusammenhang mit der Thema/Rhema-Struktur des Satzes. Insbesondere mit Bezug auf RV, in denen "nachgeholte' Information vermittelt wird, die einem Hörer mit entsprechèndem Kontextwissen auch unterstellt werden könnte" (ebd.: 155), führt er aus, daß entgegen der heute üblichen Auffassung, im unmarkierten Fall gehe das Thema dem Rhema voraus bzw. gegebene Information oder die Exposition des Gegenstandes stehe vor der neuen Information oder der Vermittlung der wichtigen Aussage, die erste Generation der Erforscher der deutschen Umgangssprache, WEGENER (1885), H. WUNDERLICH (1894) und BEHAGEL (1927), das genaue Gegenteil feststellten: "Sie behaupten, zunächst überraschend, die umgekehrte Informationsverteilung in der deutschen Umgangssprache. (...) Alle genannten Autoren argumentieren, daß die Festlegung des Themas in umgangssprachlichen deutschen Äußerungen sehr knapp ausfallen kann bzw. oft sogar aus der allgemeinen Sprechsituation heraus so klar ist, daß keine sprachliche Themeneinführung notwendig ist. (...) Erst im Nachhinein wird es für den Sprecher unter Umständen notwendig, weitere thematische Information nachzuschieben, und zwar dann, wenn das Verhalten des Rezipienten dies erfordert. (...) An der Oberfläche ergibt sich dann die genaue Umkehrung der Thema/Rhema-Ordnung: Statt eines nicht versprachlichten, weil situativ unterstellbaren Themas steht am Äußerungsanfang das Rhema, dem gegebenenfalls thematische Erläuterungen folgen.

(...) Das geschilderte Verfahren des nachgeholten Themas fügt sich perfekt in die (...) Aushandelbarkeit des Endes eines Sprecherbeitrags ein. Während sich nämlich die sprachliche Einführung des Themas am Satzanfang, wo das Rederecht fest in der Hand des Sprechers ist, dem Eingriff des Rezipienten weitgehend entzieht, ist das Ende des Satzes (...) für Eingriffe des Rezipienten maximal offen. Wieweit thematisches versprachlicht wird, wird also durch denjenigen kontrolliert, für den dies geschieht - für den Hörer, der dem Sprecher unmittelbare Rückmeldung darüber gibt, ob dieser ihn unterschätzt oder überschätzt, d.h. zuwenig oder zuviel gemeinsames Wissen voraussetzt" (AUER 1991: 154). ${ }^{7}$

Die Hypothese erscheint mir plausibel, daß die Art, wie dieser Rand konkret ausgestaltet wird und wer die Initiative dazu ergreift, sich nach der prosodischen Integration oder Selbständigkeit der Rechtsexpansion unterscheidet. Im folgenden möchte ich wiederum anhand exemplarischer Beispiele zeigen, daß und in welcher Weise die beiden hier unterschiedenen Nachstellungskonstruktionen auf unterschiedliche Funktionen im Gespräch zugeschnitten sind. 
(a) "Echte" Rechlsversetzung:

Im folgenden Beispiel wird mit der RV an dieser STIFtungsgründung der Gesprächsgegenstand, auf den mit daran bereits Bezug genommen wurde, expliziert und damit offenbar das Verständnis der Rezipienten gesichert.

(30) K5:

$703 \mathrm{E}: \quad$ WAS is eigentlich so
$\mathrm{F}(/$

$704 \mathrm{E}:$ sckwrErich daran an dieser STIFtungsgründung

* 1 (

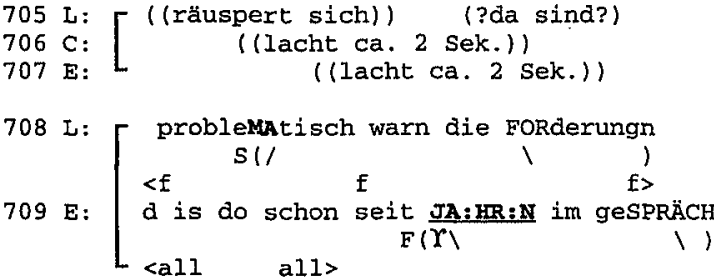

Es war zwar zuvor von der Stiftung bereits geredet worden, aber nicht von den Schwierigkeiten der Stiftungsgründung. Deshalb wäre der Satz ohne die RV nur bedingt verständlich, obwohl allen Gesprächsteilnehmern die angesprochenen Schwierigkeiten der Stiftungsgründung auf dem Hinter

grund der damals aktuellen politischen Diskussion bekannt sind. Mit der am Satzende formulierten RV wird hier also eine im Prinzip bekannte Referenz "en passant" und prospektiv vor einer möglichen Manifestation eines Verstehensproblems der Rezipientinnen gesichert.

In meinem Korpus erfüllen "echte" RV i.d.R. "en passant" verständigungssichernde Funktionen, eventuell kommen sie auch der Fremdinitijerung einer Reparatur zuvor, in einigen Fällen verlängern sie vielleicht die Einheit, um dem Sprecher Zeit für die Planung der eigenen Fortsetzung zu verschaffen. In der Mehrzahl der Fälle setzt der Sprecher selbst, ggl. nach kurzer Reparatur-Nachfrage des Rezipienten, fort, z.B. mit einer begonnenen Erzählung. Auch in unserem Beispiel setzt $E$ simultan mit $L$ ihren Turn noch fort. Die Formullerung einer RV mit einer weiteren Akzentdomãne am Satzende scheint in den meisten Fällen als Strategie zur Schaffung einer weiteren Fokusdomäne und damit eines mehrteiligen Fokus zu dienen: obwohl der primäre Akzent und Fokus sich weiter vorne findet, folgt mit der RV ein weiterer Fokus, i.d.R. mit bereits thematischem Material. Die Verständigungssicherung erfolgt unauffällig und nebenbei, d.h. "en passant" und nicht exponiert.

Dagegen liegen in den Beispielen mit einheiten-internen Pausen Fälle vor, in denen der Sprecher offenbar eine Rezipientenreaktion, wenigstens ein Rezeptionssignal, enwartet hat, dies aber ausbleibt. Das zeigt besonders deutlich das Beispiel (31): 
(31) K4: 611f

$611 \mathrm{E}$ : du wryiel leute warn denn $D A^{*} \ldots \ldots \mathrm{m}$ bei dieser

* M(I

612 EachbereichsöFFnung

1,

613

L: war ziemlich MICKrig ne

Nachdem bereits seit längerer Zeit über die Fachbereichseröffnungsfeier geredet worden ist, fragt nun $E$ ihre Kollegin $L$, wieviele Leute denn dort waren. Mit Ende der Frage WIEviel leute warn denn $D A^{*}$ wäre hier eine Turnübernahme und Antwort von $L$ fällig gewesen. $D a L$ aber schweigt, vermutet offenbar $E$ ein Verstehensproblem und expliziert die Referenz ihres vorher verwendeten Pronomens DA mit der vollen PP bei dieser fachbereichsÖFFnung. Im Unterschied zu den im folgenden beschriebenen RV-NT kann hier E offenbar, weil L ja außer Schweigen noch nichts produziert hat, die Fortsetzung auch nach dem Schweigen immer noch wie eine die vorherige Einheit einfach fortsetzende "echte" RV anhängen, an die dann $L$ sofort im Anschluß auch die enwartete Antwort liefert. Auch hier wird also dem Anschein nach nur eine weitere Fokusdomäne mit einer bereits thematischen Angabe angehängt. Dem erweckten Anschein nach wird auch diese RV also als "en passant" Verständigungssicherung hingestellt, nicht als Problembearbeitung.

\section{(b) Rechtsversetzungs-Nachträge:}

Gegenüber "echten" RV scheinen RV-Nachträge (RV-NT) vieltältiger verwendet zu werden. RV-NT sind gegenüber den prosodisch integrierten RV selbständiger: sie ersetzen oder ergänzen vorherige Bezugselemente in selbständiger Einheit und sind deshalb auch exponierten Reparaturen und Problembearbeitungen ähnlicher (vgl. JEFFERSON 1983).

Je nach der "Dringlichkeit" der Expansion für den Sprecher selbst könnte hier die Art des Anschlusses dazu dienen, sich selbst das Rederecht, z.B. für eine selbstinitierte Selbstreparatur (SCHEGLOFF/JEFFERSON/SACKS 1977), zu sichern oder Zeit für Rezipientenreaktionen einzuräumen. Während die ohne Verzögerung im Anschluß an den Satz formulierten "echten" RV mit der "en passant" Verständigungssicherung zu tun haben, bleibt bei den RV-NT oft auch mehr Zeit zwischen Vorgängersatz und NT, in der der Rezipient den NT ggf. auch veranlaßt, an ihm mitwirkt oder inn selbst produziert. Für den Sprecher ist damit die Möglichkeit gegeben, neben der Durchführung einer selbstinitiierten oder fremdinitierten Selbstreparatur auch seinen Turn zu einem weiteren möglichen Turnendepunkt zu bringen und damit eine zuvor fehlgeschlagene Turnvergabe erneut zu versuchen. Für den Rezipienten bieten RV-NT die Möglichkeit einer exponierten und expliziten Verständigungssicherung und/oder Problembearbeitung (Fremdinitiierung einer Reparatur nach SCHEGLOFF/JEFFERSON/SACKS 1977; vgl. das Beispiel (29)).

Die 19 Beispiele für RV-NT in meinem Korpus werden für folgende Funktionen verwendet: 7 im Zusammenhang mit exponierter Verständigungssicherung, 7 bei Problemen mit der Übergabe des Turns an den Rezipienten, und 5 als andere Formulierungsstrategie wie z. B. Fokussierung, 
Portionierung $0 . a ̈ ., 2$ für die Signalisierung manifester Verständigungsprobleme. Die beiden häufigsten Verwendungsweisen sollen kurz exemplifiziert werden.

(32) zeigt die Verwendung eines RV-NT zur exponierten Verständigungssicherung:

(32) KO: $1797 \mathrm{ff}$

$1798 \mathrm{D}:$ der is h:a:1:t: auch VerHaLTnis: äh.

1799 wie heiBt das.

$<p \quad p>$

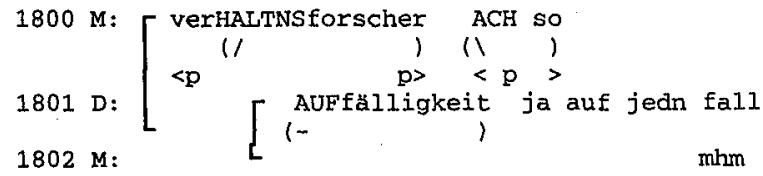

1802 D: $\Gamma$ irngdwie DAmit beschäftigt ER sich ne

* $1803 \mathrm{M}:\left\{\begin{array}{ccc}\text { irngawie Damit beschaftigt } & \text { ER sich } \\ & \text { minm }\end{array}\right.$

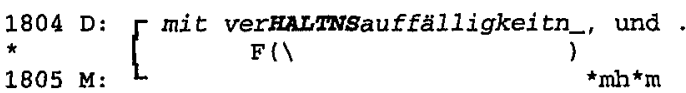

Nach vorausgegangenen Formulierungsproblemen formuliert D nun zuerst den Satz auf jedn fall irngdwie DAmit beschäftigt ER sich ne, fügt aber den gefundenen Begrift in dem RV-NT mit verHALTNSauffälligkeitn zwecks Verständigungssicherung noch einmal in korrekter Form an, obwohl die Rezipientin mit $A C H$ so und $m h m$ ihr Verstehen und ihre Rezeptionsrolle bereits mehrfach bestätigt hat. Danach tährt $D$ mit ihrer Erzählung fort.

Eine Verwendungsweise, in der die Formulierung eines Nachtrags als "Technik der Einklagung einer Rezipientenreaktion", manchmal bis hin zur "Technik des zweiten Versuchs der Turnübergabe", verwendet wird, zeigt das Beispiel (33):

(33) $\mathrm{K} 1$ :

$138 \mathrm{~N}$ : NICH nur DAS sondern da FINde die sin.. F() 1$)$ F()

139 inzwischen sehr unkritisch gewordn.

$140 \mathrm{~N}$ : FRAUN aso sich sELber gegenuber=das is

* T(I) <all> M(

$141 \mathrm{I}:$

$142 \mathrm{R}$ : JAa $\mathrm{M}(\backslash /)$

((ein)) ach so JA

$((\mathrm{atm})) \quad M(\backslash) M(\backslash)$
$<f$ f> $<\mathrm{f}>$ 
Nach einer Kritik ihrer Gesprächspartnerin I am Verhalten von Frauen in Frauenseminaren bringt N in Zeile 138-139 einen weiteren Kritikpunkt an Frauen ins Gespräch ein. Nachdem eine Rezipientenreaktion im Anschluß an ihren Satz ausgeblieben und eine kurze Pause verstrichen ist, fügt $N$ nun die Nachtrags-RV FRAUN an, die die Referenz des referierenden Pronomens die aus dem Vorgängersatz expliziert. Damit wird einerseits das Verständnis der Rezipienten gesichert, andererseits bringt $\mathrm{N}$ aber auch ihren Satz in neuer Einheit zu einem weiteren Abschlußpunkt und klagt eine $\mathrm{Re}$ zipientenreaktion ein, die dann mit l's $J A a$ ja auch gegeben wird. Sofort im Anschluß fügt $N$ noch einen weiteren Nachtrag an: aso sich SELber gegenüber. Im Anschluß hieran reagiert nun auch $R$ mit ach SO JA. Hiermit signalisiert $R$ einerseits die Lösung eines Verstehensproblems. Andererseits unterbricht er jedoch N's gerade begonnenen neuen Satz mit lauter Stimme und kämptt hier und in den Folgezeilen um sein von ihm als legitim beanspruchtes Rederecht (FrENCH/LOCAL 1983, SELTING 1991). Dies legt nahe, daß er N's Nachträge nach ihrem Satz als Techniken interpretiert hat, das Rederecht abzugeben und einen Beitrag der Rezipienten einzuklagen, den er ja nun zu geben bereit und willens ist und deshalb auch dafür das Rederecht beanspruchen kann.

Meine Daten legen nahe, daß die meisten der RV-NT nach syntaktisch vollständigen Sätzen etwas mit der Sprecher-Hörer-Koordination im Hinblick auf exponierte Verständigungssicherung und/oder die Organisation des Sprecherwechsels zu tun haben. Im Unterschied zu den "echten" RV, die v.a. als Strategie zur Schaffung eines mehrteiligen Fokus analysiert wurden und meist prospektiv und "en passant" verständigungssichernde Funktionen erfüllen, etablieren die RV in selbständiger prosodischer Einheit einen eigenständigen Fokus, der die Aufmerksamkeit des Rezipienten auf die mit dem RV-NT etablierte konversationelle Aktivität lenkt, nämlich v.a. exponierte Verständigungssicherung, Problembearbeitung und Aushandlung der Turnverteilung.

\subsection{Die Flexibilität von $X P n$ zwischen Sätzen}

Als Fazit dieser Strukturanalyse der Herausstellungen aus dem Satz ergibt sich folgendes: Weder der Satzanfang noch das Satzende sind starre Strukturen, sondern an beiden Rändern können Phrasen voran- oder nachgestellt werden. Diese Phrasen stehen vor, nach bzw. oft auch zwischen syntaktisch möglichen bzw. vollständigen Sätzen. Mit Hilfe ihrer morphologischen Markierung zeigen sie ihre Bezogenheit auf inren Bezugssatz an. Daß auch diese aber flexibel in den Formulierungsprozeß eingebunden werden können, zeigt folgendes Beispiel:

\section{(34) $\mathrm{BB} 7 / 5 \mathrm{~B}$}

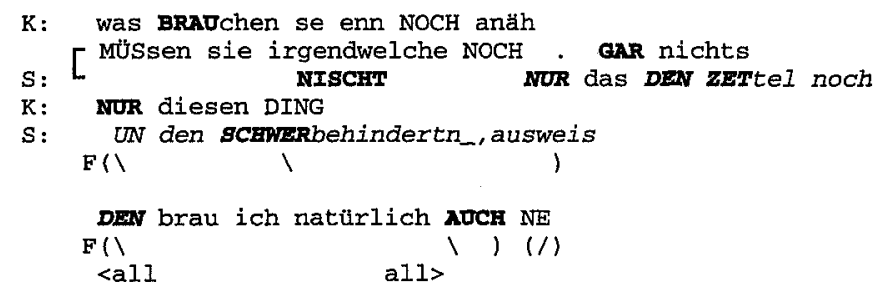


Hier wird die Phrase UN den SCHWERbehindertnausweis zunächst als Nachtrag zur Antwort NUR das DEN ZETtel noch formuliert, aber dann retrospektiv durch ihre Wiederaufnahme im Folgesatz DEN brau ich natürlich AUCH zum Freien Thema umfunktioniert. Dies zeigt, daß prosodisch selbständige Phrasen mit Hilfe ihrer morphologischen Markierung und der möglichen Wiederaufnahme in beide Richtungen mit Nachbarsätzen kohäsiv verknüpfbar sind.

\section{Fazit}

Die Morpho-Syntax und die Prosodie werden als je eigenständige Konstruktionsschemata verwendet, die jedoch systematisch zu Merkmalsbündeln kombiniert werden, um sprachlich wohl unterschiedene Konstruktionen zu erzeugen. Die Prosodie leistet dabei u.a. die Segmentierung des Redestroms in Einheiten und ermöglicht die unterschiedliche Verwendbarkeit ansonsten syntaktisch identischer Syntagmen in Gesprächen. Die Morpho-Syntax signalisiert dann die Beziehungen innerhalb von prosodisch signalisierten Einheiten oder die Beziehungen benachbarter prosodisch abgegrenzter Einheiten zueinander, u.a. die Beziehung von randständigen Phrasen zu ihren Nachbarsätzen. Prosodisch integrierte Herausstellungen weisen auf satzsyntaktische Beziehungen hin, prosodisch selbständige Herausstellungen auf diskurssemantische.

Ich habe zu zeigen versucht, daß solche Konstruktionen an den Satzrändern, Voranstellungen und Nachstellungen von Phrasen vor bzw. nach dem Satz, die morpho-syntaktisch auf Nachbarsätze bezogen und prosodisch integriert oder selbständig formuliert werden können, als systematische Ressource der Gesprächsorganisation dienen. Das Zusammenspiel prosodischer und syntaktischer Signalisierungsmittel konstituiert je unterschiedliche sprachliche Konstruktionen, die auf die Erfüllung je spezifischer kommunikativer Aufgaben im Gespräch zugeschnitten sind.

Da interaktive Aufgaben und deren Lösung nicht determiniert sind, sondern sich in der lokalen Interaktion zwischen Sprecher und Hörer situations- und rezipientenbezogen stellen, kann man also nicht behaupten, Wortstellung sei durch die Interaktion determiniert. Dennoch sind zumindest die von mir betrachteten Konstruktionen genau auf die Lösung spezifischer interaktiver Aufgaben zugeschnitten und ihre je unterschiedlichen Formen und Strukturen können in diesem Sinne rational erklärt werden. 


\section{Anmerkungen}

${ }^{1}$ Für eine Beschreibung meines Korpus und meiner Transkriptionskonventionen siehe SELTING (1991, 1993a).

2 Eine ausführlichere Analyse dieser Konsiruktionen erscheint als SELTING (1993a). Der Untersuchung lagen $16 \mathrm{LV}$ und $32 \mathrm{FT}$ zugrunde.

3 Zu LVn vor anderen Satztypen als Verb-Zweit-Sătzen, wo dann die Wiederaufnahme im Mittelfeld erfolgt, siehe Cardinaletti (1987).

4 Im Unterschied zu ALTMANN (1981) analysiere ich "Parenthesenischen" nicht als Evidenz, daß die entsprechende Konstruktion in einen sie umgebenden Satz eingebettet sein muß und deswegen ein Hinweis auf das Vorliegen einer Linksversetzung im Vorfeld eines Satzes ist. Das Vorkommen von Parenthesen ist in meiner Perspektive nicht beschrānkt auf Sätze, sondern auf projektierte Turnkonstruktionseinheiten. Deshalb können Parenthesen durchaus auch nach Freien Themen stehen, nicht nur nach Linksversetzungen.

5 Die vorangesteltten und später wiederaufgenommenen Phrasen sind v.a. Subjekte oder Objekte der Folgesätze. Da aber Subjekte und zwecks Kohärenzherstellung topikalisierte Phrasen nicht unbedingt auch einen Fokus bilden müssen, schafft gerade für solche Konstituenten die Voranstellung erst eine Möglichkeit der markierten Topikalisierung, die im Rahmen des einfachen Satzes gar nicht zur Verfügung stünde und die gerade deshalb die Interpretation einer Fokussierung auf Gesprächsebene erzeugt. Dann wäre die Voranstellung eine Möglichkeit, Phrasen auf der gesprăchssemantischen Ebene markiert zu topikalisieren und damit zu fokussieren.

${ }^{6}$ ALTMANN (1981:55) unterscheidet diese Konstruktionstypen nicht. Er spricht nur von RV, deren Grenzen fast immer durch Pausen markiert seien. Eine genaue Beurteilung der Entsprechung zu meiner hier versuchten Unterscheidung ist jedoch nicht möglich, da ALTMANN in seinen Beispielen die AV durch ein Komma abgrenzt. Ob die angeblich nötige Pause eine an phonologischen Analysen orientierte "virtuelle" oder sogenannte "linguistische" Pause oder eine phonetisch realisierte Pause ist, wird nicht gesagt. ALTMANN (ebd.) spricht jedoch weiterhin davon, daß dem FT am rechten Salzrand eine Entsprechung fehle: "Einen Konstruktionstyp, der dem Freien Thema bei den Herausstellungen nach rechts eindeutig entspricht, gibt es nicht. Zwar gibt es einige Indizien, daß es sich bei Rechtsversetzung nicht um eine einheitliche Regel handeln kōnnte, aber sie verdichten sich nicht zu einem neuen Typ." Könnte es sein, daß dieser Befund durch die Art der herangezogenen Daten und deren Repräsentation bedingt ist? Meine eigene Untersuchung basiert auf 16 Beispielen für "echte" AV und 19 für RV-NT.

7 Dagegen ist die Information laut AuER in den meisten Fällen von Nachträgen, Ausklammerungen, reparierenden, konjunktionalen oder appositiven Expansionen nicht thematisch, sondern rhematisch. Die Stärke der Rhematizität hăngt von der prosodischen Verpackung ab: "Je stärker die prosodische Integration in das Bezugssyntagma, um so schwảcher die Rhematizităt der Expansion" (ebd.: 155).

\section{Literatur}

ALTMANN, Hans (1981): Formen der "Herausstellung" im Deutschen. Tübingen: Niemeyer

AUER, Peter (1992): The Neverending Sentence: Rightward Expansion in Spoken Language. In: Kontra, M.Náradi, T. (eds.): Siudies in Spoken Language. Budapest 1992, S. 41-59

-- (1991): Vom Ende deutscher Sätze. In: Zeitschrift für Germanistische Lingustik (ZGL) 19: $139-157$

CARDINALETII, Anna (1987): Linksperiphere Phrasen in der deutschen Syntax. In: Studium Linguistik 22: 1-30

CHAFE, Wallace L. (1976): Givenness, Contrastiveness, Definiteness, Subjects, Topics, and Point of View. In: Li, Charles (ed.): Subject and Topic. New York etc.: Academic Press, S. 25-56

CinauE, Guglielmo (1977): The movement nature of left dislocation. In: Linguistic Inquiry 8.2.: $397-412$

DURANTI, Alessandro/OcHS, Elinor (1979): Left-dislocation in Italian conversation. In: Givón, Talmy (ed.): Syntax and Semantics 12: Discourse and Syntax. New York: Academic Press, S. 377-418

FreNCH, Peter/LOCAL, John (1983): Turn-competilive incomings. In: Journal of Pragmatics 7: 17-38

GELUYKENS, Ronald (1992): From discourse process to gramimatical construction. On left-dislocation in English. Amsterdam: Benjamins 
GUMPERz, John J. (1982): Discourse Strategies. Cambridge: Cambridge University Press

HofFMANN, Ludger (Mrsg.) (1992): Deutsche Syntax. Ansichten und Aussichten. Berlin etc.: de Gruyter

JACO8S, Joachim (1988): Fokus-Hintergrund-Gliederung und Grammatik. In: Altmann, Hans (Hrsg.): Intonationsforschungen. Tübingen: Niemeyer: S. 89-134

JEFFERSON, Gail (1983): On exposed and embedded correction in conversation. In: Studium Linguistik 14: 58-68

KALLMEYER, Werner (1978): Fokuswechsel und Fokussierungen als Aktivitäten der Gesprächskonstitution. In: MeyerHermann, Reinhard (Hrsg.): Sprechen - Handeln - Interaktion. Ergebnisse aus Bielefelder Forschungsprojekten zu Textheorie, Sprechakttheorie und Konversationsanalyse. Tübingen: Niemeyer, 191241

LEVINSon, Stephen C. (1983): Pragmatics. Cambridge: University Press

OCHS, Elinor/SCHIEFFELIN, Bambi (1976): Foregrounding referents: A reconsideration of left dislocation in discourse. In: Proceedings of the Second Annual Meeting of the Berkeley Linguistics Society. California, S. 240-257

OCHS Keenan, Elinor/SCHEFFELIN, Bambi B. (1983): Foregrounding referents: a reconsideration of left dislocation in discourse. In: Ochs, Elinor/Schieffelin, Bambi B. (eds.): Acquiring Conversational Competence. Boston: Routledge \& Kegan Paul, S.158-174

SACKS, Harvey/SCHEGLOFF, Emanuel A./JEFFERSON, Gail (1974): A simplest systematics for the organisation of turntaking for conversation. In: Language 50: 696-735

SCHEGLOFF, Emanuel A./JEFFERSON, Gail/SACKS, Harvey (1977): The preference for self-correction in the organisation of repair in conversation. In: Language 53: 361-382

SelTING, Margret (1991): Prosodie im Gespräch. Aspekte einer interpretativen Phonologie der Konversation. Habjlitationsschrift. Universität Oldenburg (erscheint demnächst: Tübingen: Niemeyer)

- (1993a): Phonologie der Intonation. Probleme bisheriger Modelle und Konsequenzen einer neuen interpretaliv-phonologischen Analyse. In: Zeitschrift für Sprachwissenschaft: 11,1:99-138

-- (1993b): Voranstellungen vor den Satz. Zur grammatischen Form und interaktiven Funktion von Linksversetzung und Freiem Thema im Deutschen. In: Zeitschritt für Germanistische Linguistik 21: 291-319

UHMANN, Susanne (1991): Fokusphonologie. Tübingen: Niemeyer

WootToN, Anthony J. (1989): Remarks on the methodology of conversation analysis. In: Roger, Derek/Bull, Peter (eds.): Conversation. Clevedon, Avon: Multilingual Matters, S: 238-258 